$\xi=-1$

\title{
A Proposed Approach for Evaluating Soils Optimum Moisture Content Arithmetically and Use Statistical Functions for Checking Method
}

\author{
Azhar sadiq yasun ${ }^{1 *}$, Jamal N. Al Abbasi \\ ${ }^{l}$ Department of Civil Engineering, College of Engineering, AL-Nahrain University, Baghdad /Iraq \\ ${ }^{2}$ Department of studies and planning,AL-Nahrain University, Baghdad, Iraq \\ *Corresponding Author Email: azhar28091983@gmail.com
}

\begin{abstract}
The processing of optimum moisture content for specific soils as indicated by ASTM D698 specifications detail relies upon developing the fitting third or second degree bend connection between dampness content versus soil dry unit weight on a fitting bend, the registered optimum moisture substance may contrast for a similar soil as for fitting bend figure and its position. The main objective of this study is to evaluate the optimum moisture content value based on computing average moisture content adapted from standard or modified Proctor compaction test trials and compared it with respect to the computing optimum moisture content using standard method. The research deals with a (52) compaction tests results with a wide range of optimum moisture content and dry unit weight to explore the relationships between them. The study also explores the maximum dry density values which versus standard optimum moisture content and average adopted moisture content. Statistical part depends on evaluating many statistical function values for standard and research method starts by evaluating significance of normality using Kolmogorov-Smirnov test. The average differences between standard optimum moisture content and an average value (this study depends) for moisture content was about $(-0.20)$ and an average of differences for dry unit weight values was $(0.261)$.
\end{abstract}

Keywords: Average Moisture Content; Kolmogorov-Smirnov; Maximum Dry Density; Optimum Moisture Content; Two Independent Samples T test.

\section{Introduction}

In 1933, Delegate demonstrated that the dry thickness of soil gotten by a given compaction exertion relies upon the measure of dampness the dirt contains amid compaction. For a given soil and a given compaction exertion, there is one dampness content called "ideal dampness content" that happens at a greatest dry thickness of the dirt. Those dampness substance both more noteworthy and littler than the ideal esteem will result in a dry thickness not exactly the greatest, (soil building: testing, plan, and remediation 2000). The research facility compaction technique is proposed to recreate the compactive exertion foreseen in the field, (MacIver and Sound 1986).

For development of roadways, air terminals, and different structures, usually important to minimal soil to enhance its quality. Delegate (1933) built up a research facility compaction test method to decide the most extreme dry unit weight of compaction of soils that can be utilized for determination of field compaction. This test is alluded to as the 'standard Delegate compaction test' and depends on the compaction of the dirt division passing No, 4 U.S. sieve,(Braja Das (2002).

The concentrate of compaction and unconfined compressive quality of sand altered by class F fly fiery remains. Compaction tests were performed with fluctuating compaction vitality $(2700 \mathrm{~kJ} / \mathrm{m} 3$ $300 \mathrm{~kJ} / \mathrm{m} 3)$, kinds of sand, and fly fiery debris content (0\% to $40 \%)$ separately utilizing test results uncovers that ideal estimation of unconfined compressive quality acquired for a sand-fly slag blend included $65 \%$ sand and $35 \%$ fly cinder. In light of the information acquired in the present examination, a straight scientific model has been created to foresee the ideal dampness substance of sand-fly fiery debris blend, ( Ashis Bera and Sourav (2015).

Ghanbari and Hamidi (2016) investigated numerical displaying of quick effect compaction in free sands by leading a three dimensional limited component show. This was utilized to mimic quick effect compaction (RIC) in free granular soils utilizing ABAQUS programming for one effect point. The conduct of soil under effect stacking was communicated utilizing a top pliancy display. $\mathrm{Nu}-$ merical demonstrating was done in Assalouyeh petrochemical complex in southern Iran to check the got outcomes. In-situ settlements per blow were contrasted with those in the numerical model. Estimations of enhancement by profundity were acquired from the in-situ standard infiltration, figure stacking, and substantial thickness tests and were contrasted and the numerical model outcomes. Shapes of the equivalent relative thickness plainly demonstrated the productivity of RIC horizontally and at certain profundity. Plastic volumetric strains underneath the iron block and the impact of RIC set demonstrated that an arrangement of 10 $\mathrm{mm}$ can be viewed as an edge an incentive for soil enhancement utilizing this technique. The outcomes demonstrated that RIC unequivocally enhanced the dirt up to $2 \mathrm{~m}$ top to bottom and normally impacted the dirt up to profundities of $4 \mathrm{~m}$.

Kok, Muhammad and Ghazali (2015) attempted to examine the connection between most extreme dry thickness and ideal dampness content and their relationship work with file properties, Extra factors are incorporated into the multi direct relapse (MLR) examinations, for example, grain estimate circulation and explicit gravi- 
ty other than the list properties. The suggested model requires just the pliancy list and explicit gravity.

ASTM D698-12 prescribed computing the dry unit weight and ideal water substance of each compacted example by plotting the qualities and draws the compaction bend as a smooth bend through the focuses. Plot dry unit weight to the closest $0.1 \mathrm{lbf} / \mathrm{ft} 3$ $(0.2 \mathrm{kN} / \mathrm{m} 3)$ and water substance to the closest $0.1 \%$. From the compaction bend, decide the ideal water substance and most extreme dry unit weight.

Talukdar and Sharma(2014) manages the assurance of compaction attributes of soil by static compaction technique they presumed that A static compaction strategy has been contrived in the research facility to decide the standard Delegate's most extreme dry thickness and the ideal dampness content. The connection between dampness substance and dry thickness acquired from static compaction test has likewise been observed to be explanatory in nature. The OMC acquired from static and standard Delegate test has been observed to be the equivalent. From the examination, a comparable static weight around of $820 \mathrm{kN} / \mathrm{m} 2$ has been gotten. This proportionate static weight can be utilized in the field to acquire most extreme dry thickness and OMC comparing to standard Delegate's test.

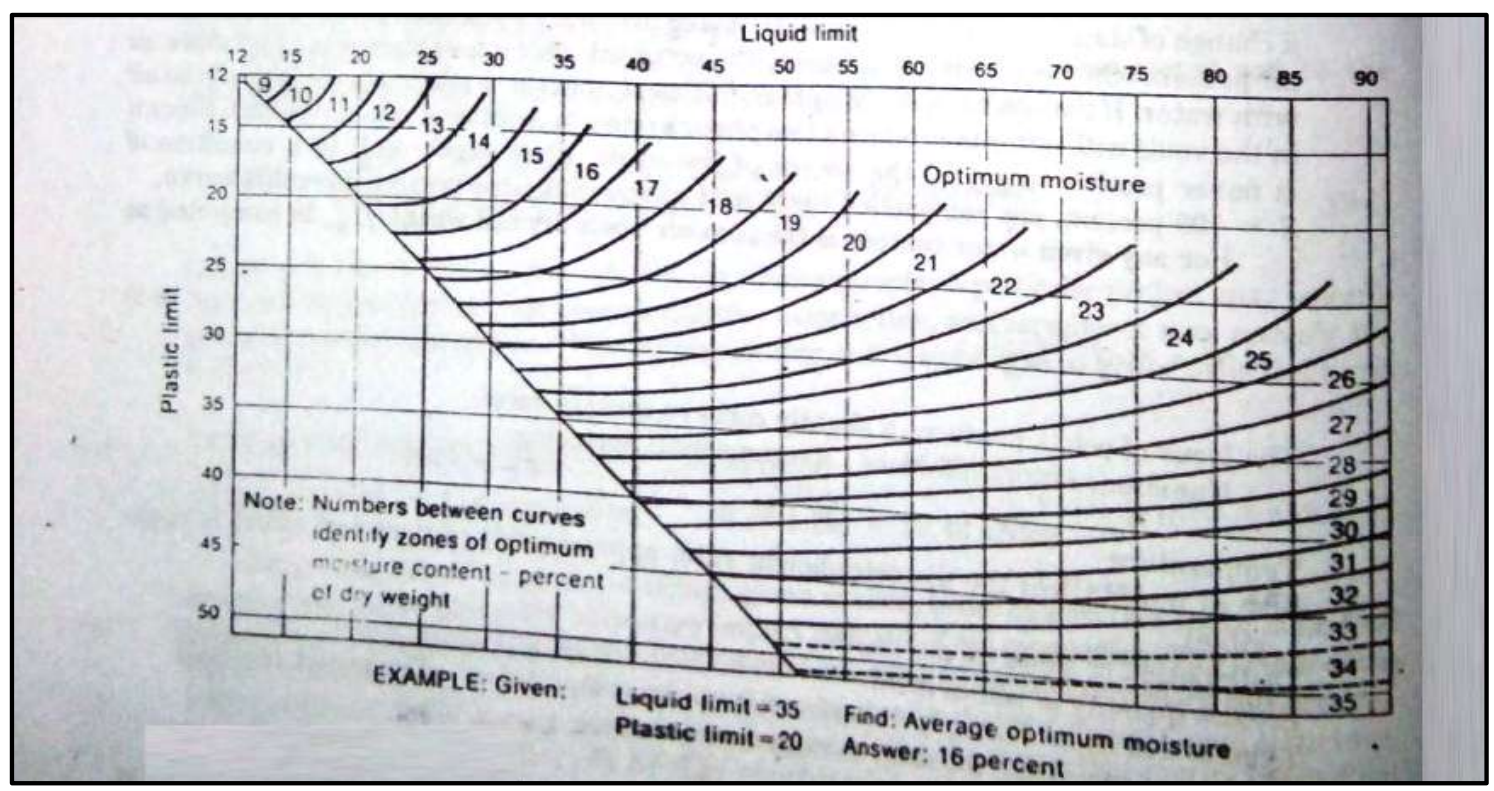

Fig. 1: Liquid limit, Plastic limit and optimum moisture content expected relationship (Joseph E. Bowles (1992)

by using the computed optimum moisture content from next study

\section{Soils used for compaction purposes grada- tion and plasticity properties}

According to the standard indexed as ASTM D698 soils can be used as a fill material to compact if it's comply with one of the three conditions below:

1. Method A in this method the soil may be used if $25 \%$ or less by mass of the material particles is retained on the No. 4 (4.75-mm) sieve.

2. Method B in this method the soil may be used if $25 \%$ or less by mass of the material particles is retained on the 3/8-in. (9.5-mm) sieve.

3. Method C in this method the soil may be used if $30 \%$ or less by mass of the material particles is retained on the 3/4-in. (19.0-mm) sieve.

Also with respect to B.S.(1377 part 4) standard there are three expected procedures for compaction as below:

1. The $2.5 \mathrm{~kg}$ rammer method used an air dried representative sample of the soil under test is passed through a 20 $\mathrm{mm}$ sieve and $5 \mathrm{~kg}$ is collected.

2. The $4.5 \mathrm{~kg}$ rammer method in this compaction test, the mold and the amount of dry soil used are the same as for the $2.5 \mathrm{~kg}$ rammer method but a heavier compaction effort is applied to the test sample.

3. The vibrating hammer method The British Standard vibrating hammer test is carried out on soil having passed the $37.5 \mathrm{~mm}$ sieve.

The study was carried out on many tests some of soils adopted as fill material in certain Iraqi projects and others was depends soils located outside Iraq, therefore and to characterize additional soil properties (liquid limit and plastic limit) the Figure-1- below can be regarded as referenced chart to explore soil plasticity behavior parts (standard type) Joseph E. Bowles (1992).

As an example the Table-1- below refer to certain data to explore soil plasticity behavior for the compacted soil samples.

Table 1:- expected L.L. and P.L. from Figure (1)

\begin{tabular}{llll}
\hline $\begin{array}{c}\text { Sample number } \\
\text { and referenced } \\
\text { Figure (number) }\end{array}$ & $\begin{array}{c}\text { Standard opti- } \\
\text { mum moisture } \\
\text { content\% }\end{array}$ & $\begin{array}{c}\text { Expected L.L. } \\
\text { from Figure(1) }\end{array}$ & $\begin{array}{c}\text { Expected P.L. } \\
\text { from Figure(1) }\end{array}$ \\
\hline 14 Figure(2) & 12.2 & 24 & 15 \\
20 Figure(3) & 15 & 35 & 13 \\
4 Figure(4) & 13 & 27 & 16 \\
34 Figure(5) & 17.2 & 40 & 21 \\
46 Figure(6) & 16.8 & 40 & 21
\end{tabular}

\section{Compaction tests result}

The main concept of soil compaction test is to compute the optimum moisture content and maximum dry unit weight of the tested soil.

The compaction test results includes (52) tests, which represent compaction curves collected for many soil types with wide range of maximum dry unit weight and optimum moisture content.

Some of the tests conducted on three trials, other tests conducted on more than three trials to compute the maximum dry unit weight and optimum moisture content. In this study different international units were used in the tests calculations.

Figures 2-7 viewed all test results that will be adopted for computing of standard optimum moisture content and maximum dry unit weight and new optimum moisture content trail values and also dry unit weight versus it. 


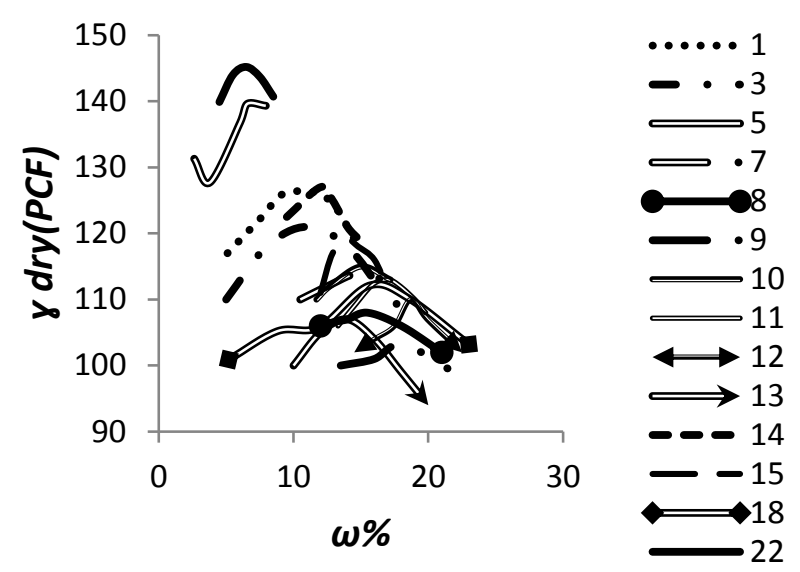

Fig. 2: Moisture content and dry unit weight relationship (tests 1, 3, 5, 7$15,18,22)$

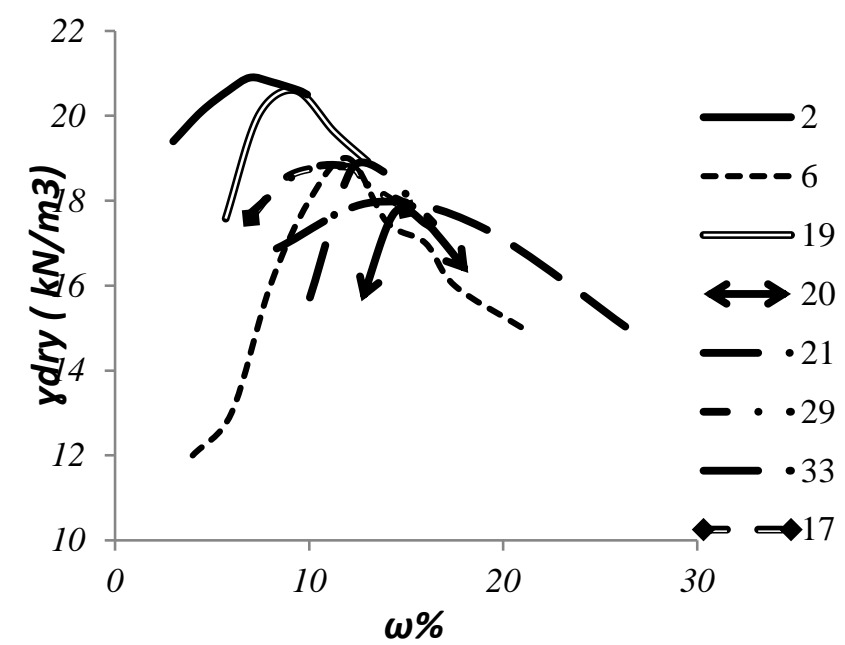

Fig. 3: Moisture content and dry unit weight relationship (tests 2, 6, 19, $20,21,29,33$ and 17)

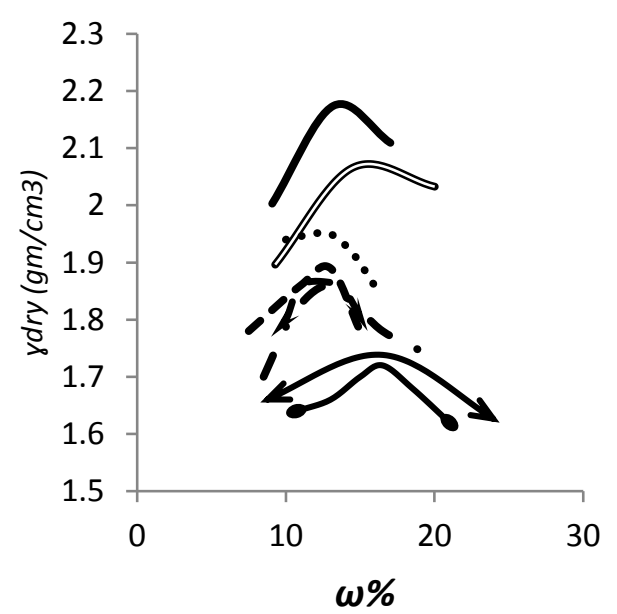

Fig.4: Moisture content and dry unit weight relationship (tests 4, 16, 23, $24,25,26,27$ and 28$)$

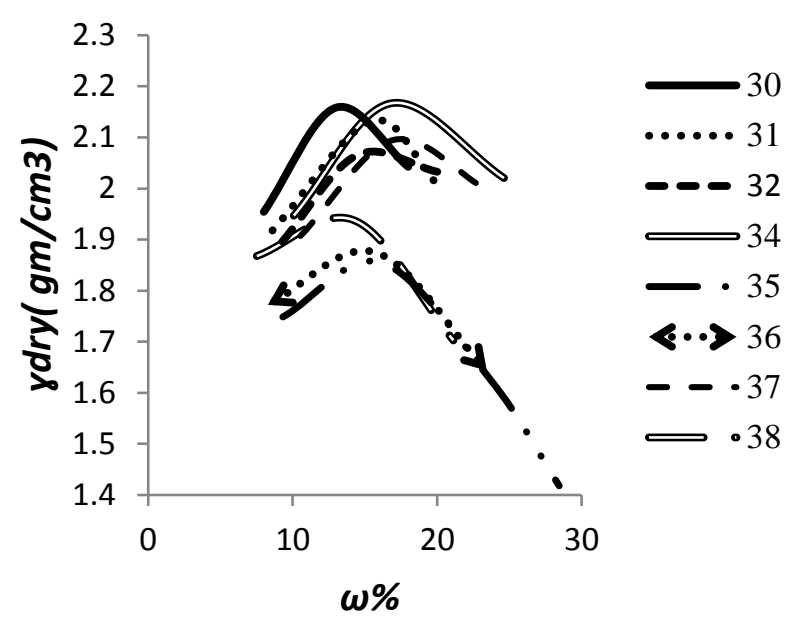

Fig.5: Moisture content and dry unit weight relationship (tests 30, 31, 32, $34,35,36,37$ and 38 )

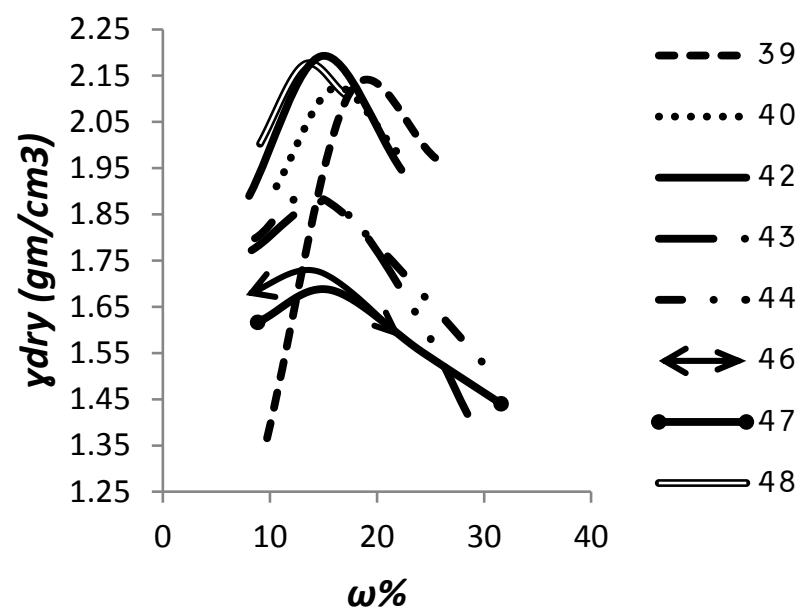

Fig.6: Moisture content and dry unit weight relationship (tests 39, 40, 42, $43,44,46,47$ and 48 )

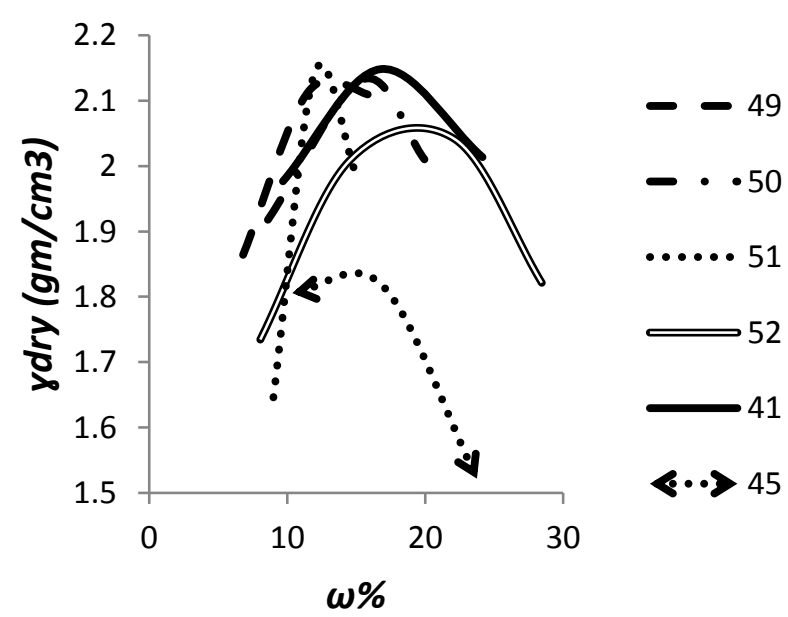

Fig.7: Moisture content and dry unit weight relationship (tests 49, 50, 51, 52, 41 and 45)

Figures 2-7 were constructed based on the unity of dry density units.

Table-2-surmised all test results that adopted for evaluation of the reality of using average moisture content as the optimum. The maximum dry densities versus standard optimum and average moisture content (study method) were also given in Table-2-. 
Table -2- Optimum moisture and dry density computing by the standard method (from top of smoothing curve) and research method (moisture depends the average of moisture content compaction trails values and dry density versus)

\begin{tabular}{|c|c|c|c|c|}
\hline $\begin{array}{l}\text { Test } \\
\text { no. }\end{array}$ & $\begin{array}{c}(\omega \%)_{\text {Opt. }} \text { from Standard } \\
\text { method }\end{array}$ & $\begin{array}{l}\text { Ydry from } \\
\text { Standard } \\
\text { conven- } \\
\text { tional } \\
\text { method } \\
(\mathrm{pcf}, \\
\mathrm{kN} / \mathrm{m}^{3} \text {, } \\
\left.\mathrm{gm} / \mathrm{cm}^{3}\right)\end{array}$ & $\begin{array}{l}\text { Average } \\
(\omega \%) \text { for } \\
\text { compac- } \\
\text { tion trails } \\
\text { for each } \\
\text { test (calcu- } \\
\text { lated } \\
\text { arithmeti- } \\
\text { cally) }\end{array}$ & $\begin{array}{l}\text { Ydry ver- } \\
\text { sus } \\
\text { average } \\
(\omega \%) \\
(\mathrm{pcf}, \\
\mathrm{kN} / \mathrm{m}^{3}, \\
\left.\mathrm{gm} / \mathrm{cm}^{3}\right)\end{array}$ \\
\hline 1 & 11 & $126.5(\mathrm{pcf})$ & 9.94 & $\begin{array}{c}126.1407 \\
977\end{array}$ \\
\hline 2 & 7 & $\begin{array}{c}20.8(\mathrm{kN} / \mathrm{m} \\
3)\end{array}$ & $\begin{array}{c}6.8085714 \\
29\end{array}$ & $\begin{array}{c}20.79916 \\
263\end{array}$ \\
\hline 3 & 11 & $121(\mathrm{pcf})$ & 11 & 120.8273 \\
\hline 4 & 13 & $\begin{array}{c}1.95(\mathrm{gm} / \mathrm{c} \\
\left.\mathrm{m}^{3}\right)\end{array}$ & 13.1 & 1.940867 \\
\hline 5 & 6.7 & $139.5(\mathrm{pcf})$ & 5.394 & $\begin{array}{c}135.5648 \\
219\end{array}$ \\
\hline 6 & 12 & $19\left(\mathrm{kN} / \mathrm{m}^{3}\right)$ & $\begin{array}{l}12.055555 \\
56\end{array}$ & 19 \\
\hline 7 & 15.2 & 114 (pcf) & 15.05 & $\begin{array}{c}113.6517 \\
663\end{array}$ \\
\hline 8 & 15.5 & $108(\mathrm{pcf})$ & $\begin{array}{c}15.583333 \\
33\end{array}$ & $\begin{array}{c}107.4987 \\
813\end{array}$ \\
\hline 9 & 17.1 & 103.8 (pcf) & $\begin{array}{c}18.083333 \\
33\end{array}$ & $\begin{array}{c}106.8766 \\
707\end{array}$ \\
\hline 10 & 15.1 & 115.5 (pcf) & 14.15 & $\begin{array}{c}114.1746 \\
168\end{array}$ \\
\hline 11 & 16.7 & 113.9 (pcf) & $\begin{array}{c}16.757142 \\
86\end{array}$ & $\begin{array}{c}112.6931 \\
211\end{array}$ \\
\hline 12 & 19.5 & 110 (pcf) & $\begin{array}{c}18.183333 \\
33\end{array}$ & 107 \\
\hline 13 & 13.9 & $107(\mathrm{pcf})$ & 15 & 105.645 \\
\hline 14 & 12.2 & 127 (pcf) & 12.66 & 126 \\
\hline 15 & 14 & 119 (pcf) & 14.45 & $\begin{array}{c}118.8458 \\
233\end{array}$ \\
\hline 16 & 12.5 & $\begin{array}{l}1.887(\mathrm{gm} / \\
\left.\mathrm{cm}^{3}\right)\end{array}$ & 11.625 & $\begin{array}{c}1.948862 \\
305\end{array}$ \\
\hline 17 & 12 & $\begin{array}{c}18.8(\mathrm{kN} / \mathrm{m} \\
\left.{ }^{3}\right)\end{array}$ & 11.2 & $\begin{array}{c}18.82098 \\
08\end{array}$ \\
\hline 18 & 15 & $104(\mathrm{pcf})$ & 13.85 & $\begin{array}{c}102.6791 \\
368\end{array}$ \\
\hline 19 & 9 & $\begin{array}{c}20.6(\mathrm{kN} / \mathrm{m} \\
3)\end{array}$ & 9.34 & $\begin{array}{c}20.55599 \\
727\end{array}$ \\
\hline 20 & 15 & $\begin{array}{c}17.9(\mathrm{kN} / \mathrm{m} \\
3)\end{array}$ & 15.6 & $\begin{array}{c}17.51899 \\
52\end{array}$ \\
\hline 21 & 13 & $19\left(\mathrm{kN} / \mathrm{m}^{3}\right)$ & 13.528 & $\begin{array}{c}18.77797 \\
162\end{array}$ \\
\hline 22 & 6.1 & 145 (pcf) & 6.5 & $\begin{array}{c}145.1165 \\
25\end{array}$ \\
\hline 23 & 12 & $\begin{array}{c}1.867(\mathrm{gm} / \\
\left.\mathrm{cm}^{3}\right)\end{array}$ & $\begin{array}{c}12.083333 \\
33\end{array}$ & $\begin{array}{c}1.869377 \\
778\end{array}$ \\
\hline 24 & 16.2 & $\begin{array}{c}1.72(\mathrm{gm} / \mathrm{c} \\
\left.\mathrm{m}^{3}\right)\end{array}$ & $\begin{array}{c}15.783333 \\
33\end{array}$ & 1.7 \\
\hline 25 & 13 & $\begin{array}{c}1.86(\mathrm{gm} / \mathrm{c} \\
\left.\mathrm{m}^{3}\right)\end{array}$ & 13.7 & $\begin{array}{c}1.936785 \\
1\end{array}$ \\
\hline 26 & 15.5 & $\begin{array}{l}2.085(\mathrm{gm} / \\
\left.\mathrm{cm}^{3}\right)\end{array}$ & $\begin{array}{l}14.600021 \\
54\end{array}$ & $\begin{array}{c}2.066024 \\
277\end{array}$ \\
\hline 27 & 13.7 & $\begin{array}{c}2.18(\mathrm{gm} / \mathrm{c} \\
\left.\mathrm{m}^{3}\right)\end{array}$ & $\begin{array}{c}13.104233 \\
33\end{array}$ & $\begin{array}{c}2.164064 \\
212\end{array}$ \\
\hline 28 & 14.6 & $\begin{array}{l}2.22(\mathrm{gm} / \mathrm{c} \\
\left.\mathrm{m}^{3}\right)\end{array}$ & $\begin{array}{c}16.390880 \\
26\end{array}$ & $\begin{array}{c}1.728956 \\
516\end{array}$ \\
\hline 29 & 11 & $\begin{array}{c}18.8(\mathrm{kN} / \mathrm{m} \\
\left.{ }_{3}\right)\end{array}$ & $\begin{array}{c}11.309673 \\
32\end{array}$ & 18.85 \\
\hline 30 & 13.5 & $\begin{array}{c}2.16(\mathrm{gm} / \mathrm{c} \\
\left.\mathrm{m}^{3}\right)\end{array}$ & 13 & 2.1583 \\
\hline 31 & 15 & $\begin{array}{l}2.138(\mathrm{gm} / \\
\left.\mathrm{cm}^{3}\right)\end{array}$ & $\begin{array}{c}14.683333 \\
33\end{array}$ & $\begin{array}{c}2.125105 \\
222\end{array}$ \\
\hline 32 & 15.8 & $\begin{array}{c}2.07(\mathrm{gm} / \mathrm{c} \\
\left.\mathrm{m}^{3}\right)\end{array}$ & $\begin{array}{c}14.600021 \\
54\end{array}$ & $\begin{array}{c}2.066024 \\
277\end{array}$ \\
\hline 33 & 15 & $18\left(\mathrm{kN} / \mathrm{m}^{3}\right)$ & $\begin{array}{c}18.641019 \\
01\end{array}$ & $\begin{array}{c}17.53860 \\
734\end{array}$ \\
\hline 34 & 17.2 & $\begin{array}{c}2.17(\mathrm{gm} / \mathrm{c} \\
\left.\mathrm{m}^{3}\right)\end{array}$ & $\begin{array}{c}17.172420 \\
4\end{array}$ & $\begin{array}{c}2.178778 \\
765\end{array}$ \\
\hline 35 & 15 & $1.86(\mathrm{gm} / \mathrm{c}$ & 16.418240 & 1.809446 \\
\hline
\end{tabular}

\begin{tabular}{|c|c|c|c|c|}
\hline $\begin{array}{l}\text { Test } \\
\text { no. }\end{array}$ & $\begin{array}{c}(\omega \%)_{\text {Opt. }} \text { from Standard } \\
\text { method }\end{array}$ & $\begin{array}{c}\text { Ydry from } \\
\text { Standard } \\
\text { conven- } \\
\text { tional } \\
\text { method } \\
(\mathrm{pcf}, \\
\mathrm{kN} / \mathrm{m}^{3} \text {, } \\
\left.\mathrm{gm} / \mathrm{cm}^{3}\right)\end{array}$ & $\begin{array}{l}\text { Average } \\
(\omega \%) \text { for } \\
\text { compac- } \\
\text { tion trails } \\
\text { for each } \\
\text { test (calcu- } \\
\text { lated } \\
\text { arithmeti- } \\
\text { cally) }\end{array}$ & $\begin{array}{c}\text { Xdry ver- } \\
\text { sus } \\
\text { average } \\
(\omega \%) \\
(\mathrm{pcf}, \\
\mathrm{kN} / \mathrm{m}^{3}, \\
\left.\mathrm{gm} / \mathrm{cm}^{3}\right)\end{array}$ \\
\hline & & $\left.\mathrm{m}^{3}\right)$ & 81 & 969 \\
\hline 36 & 15.2 & $\begin{array}{c}1.88(\mathrm{gm} / \mathrm{c} \\
\left.\mathrm{m}^{3}\right)\end{array}$ & $\begin{array}{c}15.818116 \\
57\end{array}$ & $\begin{array}{c}1.871650 \\
144\end{array}$ \\
\hline 37 & 16.8 & $2.1 \underset{3}{(\mathrm{gm} / \mathrm{cm}}$ & $\begin{array}{c}16.985042 \\
93\end{array}$ & $\begin{array}{c}2.104379 \\
583\end{array}$ \\
\hline 38 & 13.1 & $\begin{array}{c}1.94(\mathrm{gm} / \mathrm{c} \\
\left.\mathrm{m}^{3}\right)\end{array}$ & $\begin{array}{c}14.320639 \\
91\end{array}$ & $\begin{array}{c}1.933161 \\
944\end{array}$ \\
\hline 39 & 19.5 & $\begin{array}{c}2.149(\mathrm{gm} / \\
\left.\mathrm{cm}^{3}\right)\end{array}$ & $\begin{array}{c}17.569254 \\
59\end{array}$ & $\begin{array}{c}2.103963 \\
215\end{array}$ \\
\hline 40 & 16.5 & $2.1 \underset{3}{(\mathrm{gm} / \mathrm{cm}}$ & $\begin{array}{c}16.200435 \\
05\end{array}$ & $\begin{array}{c}2.119558 \\
896\end{array}$ \\
\hline 41 & 17.5 & $\begin{array}{c}2.15(\mathrm{gm} / \mathrm{c} \\
\left.\mathrm{m}^{3}\right)\end{array}$ & $\begin{array}{c}16.872019 \\
49\end{array}$ & $\begin{array}{c}2.158730 \\
165\end{array}$ \\
\hline 42 & 15 & $\begin{array}{c}2.19(\mathrm{gm} / \mathrm{c} \\
\left.\mathrm{m}^{3}\right)\end{array}$ & $\begin{array}{c}15.075607 \\
74\end{array}$ & $\begin{array}{c}2.184616 \\
355\end{array}$ \\
\hline 43 & 14.8 & $\begin{array}{c}1.88(\mathrm{gm} / \mathrm{c} \\
\left.\mathrm{m}^{3}\right)\end{array}$ & $\begin{array}{c}18.976189 \\
79\end{array}$ & $\begin{array}{c}1.850145 \\
439\end{array}$ \\
\hline 44 & 13.8 & $\begin{array}{c}1.89(\mathrm{gm} / \mathrm{c} \\
\left.\mathrm{m}^{3}\right)\end{array}$ & $\begin{array}{c}20.867275 \\
68\end{array}$ & $\begin{array}{c}1.794550 \\
846\end{array}$ \\
\hline 45 & 15 & $\begin{array}{c}1.84(\mathrm{gm} / \mathrm{c} \\
\left.\mathrm{m}^{3}\right)\end{array}$ & $\begin{array}{c}17.007030 \\
62\end{array}$ & $\begin{array}{c}1.801542 \\
346\end{array}$ \\
\hline 46 & 14 & $\begin{array}{c}1.83(\mathrm{gm} / \mathrm{c} \\
\left.\mathrm{m}^{3}\right)\end{array}$ & $\begin{array}{c}14.846719 \\
68\end{array}$ & $\begin{array}{c}1.722069 \\
359\end{array}$ \\
\hline 47 & 17 & $\begin{array}{c}1.85(\mathrm{gm} / \mathrm{c} \\
\left.\mathrm{m}^{3}\right)\end{array}$ & $\begin{array}{c}20.069660 \\
74\end{array}$ & $\begin{array}{c}1.656591 \\
526\end{array}$ \\
\hline 48 & 13.5 & $\begin{array}{l}2.175(\mathrm{gm} / \\
\left.\mathrm{cm}^{3}\right)\end{array}$ & $\begin{array}{c}13.104233 \\
33\end{array}$ & $\begin{array}{c}2.164064 \\
212\end{array}$ \\
\hline 49 & 13 & $\begin{array}{c}2.13(\mathrm{gm} / \mathrm{c} \\
\left.\mathrm{m}^{3}\right)\end{array}$ & 11.6 & 2.120524 \\
\hline 50 & 15.5 & $\begin{array}{c}2.14(\mathrm{gm} / \mathrm{c} \\
\left.\mathrm{m}^{3}\right)\end{array}$ & $\begin{array}{c}14.683333 \\
33\end{array}$ & $\begin{array}{c}2.125105 \\
222\end{array}$ \\
\hline 51 & 12.5 & $\begin{array}{c}2.16(\mathrm{gm} / \mathrm{c} \\
\left.\mathrm{m}^{3}\right)\end{array}$ & 12 & 2.1482 \\
\hline 52 & 19.5 & $\begin{array}{c}2.04(\mathrm{gm} / \mathrm{c} \\
\left.\mathrm{m}^{3}\right)\end{array}$ & $\begin{array}{c}14.884002 \\
18\end{array}$ & $\begin{array}{c}2.008549 \\
57\end{array}$ \\
\hline
\end{tabular}

Table -3- and Figure 8 showed the detail of the test number 39 results which were summarized in Table -2- also if using the figure (1).

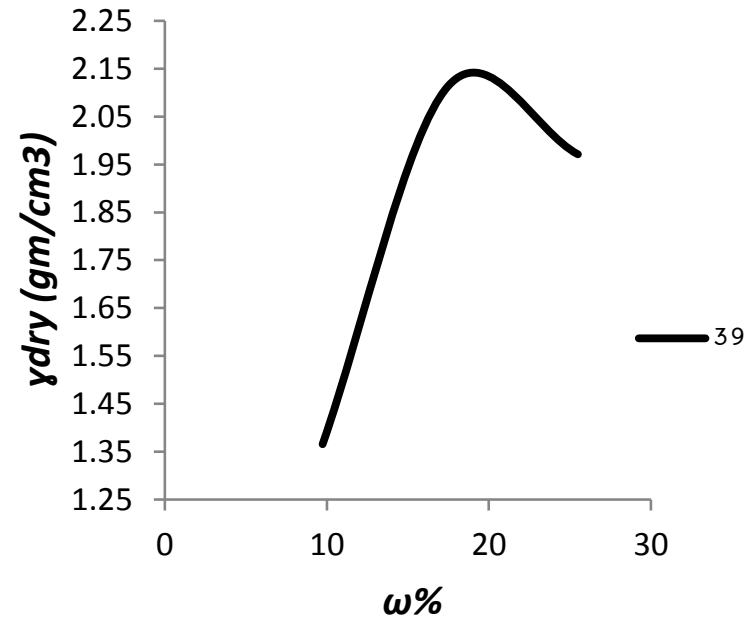

Fig.8: Moisture content and dry unit weight relationship test Number -39-

Table 3: result detail for test No.39

\begin{tabular}{rl}
\hline$\gamma_{\text {dry }}\left(\mathrm{gm} / \mathrm{cm}^{3}\right)$ & \multicolumn{1}{c}{$\omega \%$} \\
\hline 1.365535957 & 9.735839162 \\
2.113432836 & 17.4760424 \\
1.971234735 & 25.49588221 \\
Ydry maximum from curve plot & $\omega \%$ versus $\gamma_{\text {dry }}$ maximum from curve
\end{tabular}
$\left(\mathrm{gm} / \mathrm{cm}^{3}\right)$ 
2.149 $\mathrm{gm} / \mathrm{cm}^{3}$ 2.103
Ydry versus average $(\omega \%)$
19.5

Average $(\omega \%)$ for the three compaction trails 17.57
From the all above computation of average moisture content value and to build up sensible comparison between the calculated optimum moisture content using standard method and the calculated average moisture content for the same test the difference value (D.V.) is calculated as equations(1) and (2) refers.

$$
\begin{aligned}
& D . V .(\omega) \\
& =\omega_{\text {from standard method }} \\
& -\omega_{\text {average moistutre content }}
\end{aligned}
$$

$D . V .\left(\gamma_{\text {dry }}\right)=\gamma_{\text {dry from standard method }}-$

$\gamma_{\text {dry versus average moistutre content }}$

\begin{tabular}{|c|c|c|}
\hline Test no. & D.V. $\left(\gamma_{d r y}\right)$ & D.V. $(\omega)$ \\
\hline 1 & 0.35920232 & 1.06 \\
\hline 2 & 0.000837368 & 0.1914286 \\
\hline 3 & 0.1727 & 0 \\
\hline 4 & 0.009133 & -0.1 \\
\hline 5 & 3.93517806 & 1.306 \\
\hline 6 & 0 & -0.0555556 \\
\hline 7 & 0.34823375 & 0.15 \\
\hline 8 & 0.50121875 & -0.083333 \\
\hline 9 & -3.076670698 & -0.9833333 \\
\hline 10 & 1.32538325 & 0.95 \\
\hline 11 & 1.206878878 & -0.05714286 \\
\hline 12 & 3 & 1.3166667 \\
\hline 13 & 1.355 & -1.1 \\
\hline 14 & 1 & -0.46 \\
\hline 15 & 0.1541767 & -0.45 \\
\hline 16 & -0.0618623 & 0.875 \\
\hline 17 & -0.0209808 & 0.8 \\
\hline 18 & 1.32086322 & 1.15 \\
\hline 19 & 0.04400272 & -0.34 \\
\hline 20 & 0.3810048 & -0.6 \\
\hline 21 & 0.22202838 & -0.528 \\
\hline 22 & -0.116525 & -0.4 \\
\hline 23 & -0.00237778 & -0.083333 \\
\hline 24 & 0.02 & 0.41666667 \\
\hline 25 & -0.0767851 & -0.7 \\
\hline 26 & 0.018975723 & 0.8999780 \\
\hline 27 & 0.015935788 & 0.595766667 \\
\hline 28 & 0.491043484 & -1.7908803 \\
\hline 29 & -0.05 & -0.3096733 \\
\hline 30 & 0.0017 & 0.5 \\
\hline 31 & 0.012894778 & 0.3166667 \\
\hline 32 & 0.003975723 & 1.199978 \\
\hline 33 & 0.461392658 & -3.6410190 \\
\hline 34 & -0.00877877 & 0.027579 \\
\hline 35 & 0.050553031 & -1.4182408 \\
\hline 36 & 0.008349856 & -0.6181165 \\
\hline 37 & -0.0043796 & -0.185043 \\
\hline 38 & 0.006838056 & -1.2206399 \\
\hline 39 & 0.045036785 & 1.9307454 \\
\hline 40 & -0.0195589 & 0.29956495 \\
\hline 41 & -0.0087302 & 0.627980505 \\
\hline 42 & 0.005383645 & -0.0756077 \\
\hline 43 & 0.029854561 & -4.1761899 \\
\hline 44 & 0.095449154 & -7.0672757 \\
\hline 45 & 0.038457654 & -2.00703062 \\
\hline 46 & 0.107930641 & -0.84671968 \\
\hline 47 & 0.193408474 & -3.0696607 \\
\hline 48 & 0.010935788 & 0.3957666 \\
\hline 49 & 0.009476 & 1.4 \\
\hline 50 & 0.014894778 & 0.8166666 \\
\hline 51 & 0.0118 & 0.5 \\
\hline 52 & 0.03145043 & 4.6159978 \\
\hline Average & 0.26105633 & -0.192776 \\
\hline
\end{tabular}

Table 4: the difference values for dry density and moisture content

For all test trails the average values for D.V. was 0.261 and -0.192 for ydry and optimum $\omega \%$ respectively.

\section{The statistical function used in the study}

\subsection{Kolmogorov-smirnov normality test}

A one sample hypothesis test used to determine whether the population from which you draw your sample is normal.

The null hypothesis for a normality test states that population is normal. The alternative hypothesis states that the population is none normal.

This test compares the empirical cumulative distribution function of your sample data with the distribution expected if the data were normal. If this observed difference is sufficiently large, the test will reject the null hypothesis of population normality.

If the p-value of these tests is less than your chosen $\alpha$-level, you can reject your null hypothesis and concluded that the population is none normal (Michael Akritas (2016).

\subsection{Mann-whitney test}

A nonparametric hypothesis test was used to determine whether two population have the same population median (h). The function tests the null hypothesis that the two population medians are equal $\left(H: \mu_{x}=\mu_{y}\right)$. The alternative hypothesis can be left-tailed $\left(\mu_{x}<\mu_{y}\right)$, right-tailed $\left(\mu_{\mathrm{x}}>\mu_{\mathrm{y}}\right)$, or two-tailed $\left(\mu_{\mathrm{x}} \neq \mu_{\mathrm{y}}\right)$. The Mann-Whitney test does not require the data to come from normality distributed populations (Michael Akritas (2016).

\subsection{2-Sample t-test}

The hypothesis test was used for a mean of two normally distributed populations. It tests whether the difference between the means of two independent populations is equal to a target value. A hypothesis test for two populations means to determine whether they are significantly different. This procedure uses the null hypothesis that the difference between two population means is equal to hypothesized value ( $\left.\mathrm{H}: \mu_{\mathrm{x}^{-}} \mu_{\mathrm{y}}=\mu\right)$, and test it against an alternative hypothesis, which can be left-tailed $\left(\mu_{x}-\mu_{y}<\mu\right)$, righttailed $\quad\left(\mu_{x}-\mu_{y}>\mu\right), \quad$ or $\quad$ two-tailed $\left(\mu_{x}-\mu_{y} \neq \mu\right)$ (Michael Akritas (2016).

\section{Statistical function analysis results}

To compare the average moisture content with respect to optimum moisture content in standard method and also maximum dry unit weight or maximum density results; the statistical function Kolmogorov-Smirnov normality test was used for evaluating the normal distribution of the data to be tested with another suitable statistical functions, the results as seen in the Table-5-, noting that all statistical analysis done by using Statistical Package for the Social

\begin{tabular}{|c|c|c|c|}
\hline \multirow[b]{2}{*}{ Data type tested } & \multicolumn{3}{|c|}{ Kolmogorov-Smirnov } \\
\hline & Statistic & df & Sig. \\
\hline$\omega_{\text {average }}($ this study depends) & 0.083 & 52 & 0.200 \\
\hline $\begin{array}{l}\text { Ydry versus } \omega \text { average moisture } \\
\text { content (this study depends) }\end{array}$ & 0.126 & 52 & 0.038 \\
\hline$\omega_{\text {optimum }}$ by standard ASTM & 0.106 & 52 & 0.200 \\
\hline Ydry (max) by standard ASTM & 0.123 & 52 & 0.049 \\
\hline
\end{tabular}
Sciences (SPSS) software program.

Table 5: the normality test values

From the above data showed in the Table -5-the significance for both maximum dry density values for was not normal because of significant difference was less than (0.05) therefore the two independent samples Mann-Whitney nonparametric test will be adopted as shown in Table -6 . 
Table 6: The Mann-Whitney test values for Densities data

\begin{tabular}{|c|c|c|c|c|}
\hline \multicolumn{5}{|c|}{ Ranks } \\
\hline & $\mathrm{G}$ & $\mathrm{N}$ & Mean Rank & Sum of Ranks \\
\hline \multirow[t]{3}{*}{ Density } & 1.0000 & 52 & 55.03 & 2861.50 \\
\hline & 2.0000 & 52 & 49.97 & 2598.50 \\
\hline & Total & 104 & & \\
\hline \multicolumn{5}{|c|}{ Test Statistics } \\
\hline \multirow{2}{*}{\multicolumn{3}{|c|}{ Mann-Whitney U }} & & Density \\
\hline & & & & 1220.500 \\
\hline \multicolumn{3}{|c|}{ Wilcoxon W } & & 2598.500 \\
\hline \multicolumn{3}{|c|}{$\mathrm{Z}$} & & -0.855 \\
\hline \multicolumn{2}{|c|}{$\begin{array}{l}\text { Asymp. Sig. (2-tailed) } \\
\text { a. Grouping Variable: G }\end{array}$} & & & 0.393 \\
\hline
\end{tabular}

It's clear that a significant difference value in the Table-6- was greater than 0.05 .

To build up the comparison of optimum moisture content values for both two group the independent Samples T test is used because the distribution of the data above was a normal (significant difference >0.05) as Table -5-refers. Therefore as shown in Table -7the two independent samples $\mathrm{T}$ test function results was used to make the comparison (this type of test suppose that $\mathrm{H} 0: \mu 1=\mu 2$, in another word this test type depends no difference between the compared means against the alternative hypotheses Ha: $\mu 1 \neq \mu 2$ ), it should be noted that computations were done after converted some of units to the metric units system.

Table 7: 2 independent $\mathrm{T}$ test results for optimum moisture content data series

\begin{tabular}{lllrrr}
\hline & \multicolumn{5}{c}{ Group Statistics } \\
& $\mathrm{G}$ & $\mathrm{N}$ & Mean & Std. Deviation & \multicolumn{1}{c}{ Std. Error Mean } \\
\hline$\omega$ & 1.0000 & 52 & 14.080769 & 2.8755247 & 0.3987635 \\
& 2.0000 & 52 & 14.273545 & 3.2072153 & 0.4447607 \\
\hline
\end{tabular}

\section{Continued}

\begin{tabular}{|c|c|c|c|}
\hline \multicolumn{4}{|c|}{ Independent Samples Test } \\
\hline \multirow[t]{8}{*}{$\omega$} & & $\begin{array}{c}\text { Equal vari- } \\
\text { ances assumed }\end{array}$ & \\
\hline & $\mathrm{F}$ & 0.538 & Levene's Test for \\
\hline & Sig. & 0.465 & Equality of Variances \\
\hline & $\mathrm{t}$ & -0.323 & \\
\hline & df & 102 & \\
\hline & $\begin{array}{l}\text { Sig. (2- } \\
\text { tailed) }\end{array}$ & 0.748 & \\
\hline & $\begin{array}{l}\text { Mean Dif- } \\
\text { ference }\end{array}$ & -0.1927758 & T-test for Equality of \\
\hline & $\begin{array}{l}\text { Std. Error } \\
\text { Difference }\end{array}$ & 0.5973479 & Means \\
\hline 95\% Confi- & Lower & -1.3776125 & \\
\hline $\begin{array}{c}\text { of the Differ- } \\
\text { ence }\end{array}$ & Upper & 0.9920609 & \\
\hline
\end{tabular}

Noticeable that group (1) refers to the optimum moisture content computed using standard method and group (2) refers to research method values (based on obtaining average moisture content).

From the presented data, it is clear that there are no reasonable differences between all statistical function values. i.e. there is no significance differences between research and standard method number.

According to Tables 3-7, it can be observed that difference of moisture content and ydry maximum do not have wide range values and the most differences value were less than one unit.

\section{Conclusion}

1. The method of research for calculating the average moisture content of the compaction test trials can be used to provide a quick way of the optimum moisture content expectation.
2. The average difference values between the average moisture content (research method) and the standard method was -0.192 .

3. The average difference values between the ydry maximum versus average moisture content (research method) and the calculated ydry by the standard method was 0.261 .

4. All statistical functions results gave good acceptance indication between research and standard method values with no significance differences, therefore the calculated results indicated that adopting research method values can be acceptable with no reasonable difference values of standard method, that's more clearly for the differences of moisture content than the differences depends maximum dry densities value within research data limitation.

5. The research method in optimum moisture content value expectation was more valid when compaction curve figure symmetry about the optimum moisture content.

\section{References}

[1] Hua Chen, F., 2000. Soil Engineering. Testing, design and remediation, Florida: CRC Press LLC, p.68.

[2] MacIver, B.N. and Hale, G.P., 1986. Laboratory Soils Testing Change 2 (No. EM-1110-2-1906). Army Engineer Waterways Experiment Station Vicksburg Ms., Washington, D.C. 20314, p.126.

[3] Das, B.M., 2002. Soil mechanics laboratory manual. New York, USA: Oxford university press, p.81.

[4] K.S. NG, Y.M. Chew, M.H.Osman, and S.K. Mohamad Ghazali, 2015. Estimating Maximum Dry Density and Optimum Moisture Content of Compacted Soils, In: International Conference on Advances in Civil and Environmental Engineering, Penang: UiTM Pulau Pinang, pp.B1-9.

[5] Statistical Package for the Social Sciences (SPSS), (2016), New York, IBM Company.

[6] Smith, I., 2014. Smith's elements of soil mechanics. John Wiley \& Sons p434-435.

[7] K. Bera, Ashis and Chakraborty, Sourav. (2015). Compaction and Unconfined Compressive Strength of Sand Modified by Class F Fly Ash. Geomechanics and Engineering, An Int'l Journal Vol. 9 No.2.

[8] ASTM Committee D-18 on Soil and Rock, 2007. Standard Test Methods for Laboratory Compaction Characteristics of Soil Using Standard Effort (12 $400 \mathrm{Ft}-\mathrm{lbf} / \mathrm{ft} 3(600 \mathrm{KN}-\mathrm{m} / \mathrm{m} 3))$. ASTM International.

[9] Bowles, J.E., 1992. Engineering properties of soils and their measurement. McGraw-Hill, Inc.

[10] Talukdar, P., Sharma, B. and Shridharan, A., 2014. Static Method to Determine Compaction Characteristics of Soils.

[11] Akritas, M.G., 2016. Probability \& statistics with R for engineers and scientists. Boston, MA: Pearson. 